\title{
PREVALENCIA DEL CONSUMO DE SUSTANCIAS LICITAS E ILÍCITAS EN UNA FACULTAD DE SALUD DE UNA UNIVERSIDAD PÚBLICA DE COLOMBIA
}

Jaime Horacio Toro Ocampo ${ }^{1}$, Yolanda Restrepo Suarez ${ }^{2}$, Luz Ever Díaz Monsalve ${ }^{3}$

\section{Resumen}

Objetivo: describir la prevalencia de consumo de sustancias licitas e ilícitas en estudiantes de una facultad de salud, de una Universidad pública. Metodología: estudio descriptivo, transversal, desarrollado con 252 estudiantes a quienes se les aplicó una encuesta compuesta por tres instrumentos, versión para auto-aplicación. Resultados: las sustancias que consumían al momento de la encuesta fueron en su orden, alcohol (75.8\%), tabaco (14.3\%), marihuana (6\%), cocaína (1.2\%). Se encontró que el (53.6\%) consumió algún tipo de sustancia antes de ingresar a la universidad. La mayor motivación para el consumo fue por gusto. Por efectos de la sustancia ha tenido problemas disciplinarios el (7.9\%) y el (16.3\%) ha pensado y/o intentado suicidarse. Discusión: la representación social del consumo puede influir en la frecuencia del consumo, quizás es por ello que el alcohol sea la sustancia que más se consume, seguido del tabaco y la marihuana, en menor proporción, debido posiblemente a la estigmatización social que produce su consumo.

Palabras clave: efectos de drogas, abuso de sustancias, alcoholismo.

1 Enfermero, Magíster en Epidemiología. Docente investigador, Facultad de Enfermería, Universidad de Antioquia. Correo electrónico: horacio.toro@udea. edu.co

${ }^{2}$ Enfermera, Especialista en Drogodependencia. Docente investigadora, Facultad de Enfermería, Universidad de Antioquia. Correo electrónico: delacruz.restrepo@ udea.edu.co

${ }^{3}$ Enfermera, Especialista en Epidemiologia. Magíster en Salud Colectiva. Docente investigadora, Facultad de Enfermería, Universidad de Antioquia. Correo electrónico: ledmonsalve@gmail.com 


\section{PREVALENCE OF THE CONSUMPTION OF LEGAL AND ILLEGAL SUBSTANCES IN A MEDICINE FACULTY OF A PUBLIC UNIVERSITY OF COLOMBIA}

Jaime Horacio Toro Ocampo ${ }^{1}$, Yolanda Restrepo Suarez ${ }^{2}$, Luz Ever Díaz Monsalve ${ }^{3}$

\section{Abstract}

Objective: To describe the prevalence of consumption of legal and illegal substances in students of a medicine faculty, of a public University. Methodology: A descriptive, cross-sectional study was carried out with 252 students to whom a survey composed by three instruments was applied, version for self-study. Results: the substances that were consumed at the time of the survey were in their order, alcohol (75.8\%), tobacco (14.3\%), marijuana (6\%), cocaine(1.2\%). It was found that (53.6\%) consumed some type of substance before entering university. Pleasure was the greatest motivation for consumption. Due to, substance effects the (7.9\%) has had disciplinary problems and the $(16.3 \%)$ has thought and / or tried to commit suicide. Discussion: The social representation of consumption can influence the frequency of consumption, perhaps that is why alcohol is the most consumed substance, tobacco and marijuana in a less proportion, possibly due to the social stigmatization that produces their consumption. 


\section{PREVALÊNCIA DO CONSUMO DE SUBSTÂNCIAS LÍCITAS E ILÍCITAS EM UMA FACULDADE DE SAÚDE DE UMA UNIVERSIDADE PÚBLICA COLOMBIANA}

Jaime Horacio Toro Ocampo ${ }^{1}$, Yolanda Restrepo Suarez ${ }^{2}$, Luz Ever Díaz Monsalve ${ }^{3}$

\section{Resumo}

Objetivo: descrever a prevalência de consumo de substâncias lícitas e ilícitas entre estudantes de uma faculdade de saúde, de uma universidade pública. Metodologia: um estudo descritivo transversal desenvolvido com 252 estudantes que contestaram um questionário composto por três instrumentos, versão auto-aplicável. Resultados: substâncias consumidas no momento do levantamento foram, na seguinte ordem, álcool (75,8\%), tabaco (14,3\%), maconha (6\%), cocaína $(1,2 \%)$. Verificou-se que $(53,6 \%)$ consumiram algum tipo de substância antes de ingressar na universidade. A maior motivação para o consumo foi por prazer. Devido aos efeitos da substância $7,9 \%$ teve problemas disciplinares e $16,3 \%$ pensou e / ou tentou cometer suicídio. Discussão: A representação social de consumo pode influenciar a frequência de consumo, talvez por isso o álcool é a substância mais consumida, tabaco e marijuana são consumidos em menor grau, possivelmente por causa do estigma social que produz o seu consumo. 


\section{Introducción}

El consumo de sustancias licitas e ilícitas se ha convertido en los últimos años en un problema de salud pública, este viene afectando a la población general y en mayor medida a los jóvenes y adolescentes. Según la Oficina de las Naciones Unidas Contra las Drogas y el Delito (UNODC), se calcula que 1 de cada 20 adultos, es decir, alrededor de 250 millones de personas de entre 15 y 64 años, consumieron por lo menos una droga en 2014, con efectos devastadores para la salud (1).

El uso y abuso de substancias psicoactivas, tanto licitas como ilícitas, fomentan desde problemas de salud físicos y mentales, hasta violencia y falta de seguridad social, entre otros. Estudios realizados en Antioquia entre 2003 y 2007 revelaron que las sustancias que se consumen en el país son las mismas, y que las cifras de riesgo de dependencia al alcohol se incrementaron 9 puntos porcentuales durante ese periodo en los jóvenes escolarizados del departamento (2).

Tanto el consumo de sustancias como la clase social son determinantes de salud muy importantes, puesto que se relacionan con condiciones de vida, acceso a recursos, y problemáticas como el desempleo, la malnutrición y la carencia de vivienda adecuada, entre otras. Las personas que viven en condiciones de pobreza son más vulnerables a sufrir problemas de salud mental (3).

Las consecuencias que se relacionan, directa e indirectamente, con el consumo y adicción a sustancias en los consumidores excesivos son a menudo síntomas de disfunción de los roles familiares, como malas relaciones de pareja, violencia doméstica, maltrato infantil, negligencia de cuidados; económicos; escolares; laborales, como el ausentismo y la baja productividad; y comunitarios, entre los que se destacan ruidos, molestias, robos, peleas y conductas sexuales violentas $y$ no protegidas (4).

El consumo excesivo de una sustancia también puede causar estados de intoxicación en el organismo y convertirse en una situación grave; en un estudio realizado en una institución de salud de Medellín se encontró que, tanto la intoxicación alcohólica como la intoxicación por consumo de una o más sustancias, fueron los diagnósticos médicos más frecuentes en los pacientes atendidos en la unidad de urgencias de la institución, en una población de consumidores de sustancias psicoactivas, en un rango de edad entre los 12 y los 73 años. Estos correspondieron a un triage de $1 \mathrm{y}$ 2 , donde los pacientes consultaron por molestias gastrointestinales, estado de intoxicación por sustancias, deshidratación, taquicardia y ansiedad (5).

El consumo de sustancias psicoactivas en cualquiera de los integrantes de la familia genera impacto negativo por las consecuencias adversas que esto puede generar en el otro, entre las que se destacan el desacato a las normas establecidas por la familia, las dificultades en la comunicación, el incumplimiento o deserción de las actividades académicas o laborales, y la posibilidad de enfermar o morir. También se pueden ver reflejadas la violencia intrafamiliar, el maltrato y la separación conyugal (6). 
Algunos autores definen el consumo de sustancias "como el acercamiento tanto ocasional como habitual del sujeto con la sustancia que produce algún tipo de alteración en el sistema nervioso central" (7).

La característica social para que se incremente el uso de sustancias en ambientes universitarios tiene que ver con las posibilidades de acceso a la compra de bebidas embriagantes, tabaco $u$ otras sustancias. En estos escenarios se presenta menor control sobre el tiempo de los jóvenes por parte de los padres y se incrementan las interacciones culturales y sociales. Lo anterior, tiene un impacto directo en estos jóvenes, de modo tal que estos ambientes funcionan como espacios de iniciación en el consumo de sustancias psicoactivas o de fomento del uso recurrente de las mismas, que se enmarca en la necesidad de aceptación social de los jóvenes (8).

Los patrones de consumo se definen de acuerdo con la frecuencia de uso de la sustancia. El consumo es experimental cuando la persona prueba una o varias veces la sustancia, pero no la vuelve a consumir; la motivación de este consumo puede darse por curiosidad, por sentirse parte de un grupo, por presión de pares, por conocer nuevos amigos y/o por sentirse adulto (9). El consumo ocasional se presenta esporádicamente y se caracteriza porque el individuo sabe cuál es el tipo de droga que consume y donde puede usarla, sin que aparezcan síntomas de tolerancia y/o de abstinencia; el consumo ocasional es motivado por la búsqueda de mayor rendimiento académico, físico o sexual (9). Por otra parte, se considera consumo habitual el uso diario de las sustancias y cuando aparecen síntomas de tolerancia a estas; este tipo de personas dedican la mayor parte del tiempo a pensar en la droga, en cómo obtenerla y donde consumirla (9).

En Colombia se han llevado a cabo importantes estudios sobre la epidemiologia del consumo, el abuso y la dependencia a las sustancias, con el fin de estimar, entre otros elementos, la magnitud del consumo y el comportamiento de los trastornos por abuso y dependencia (10).

En la Encuesta Nacional de Salud Mental (11) se encontró que los trastornos por uso de alcohol en vida son del $45.8 \%$, siendo el sexo masculino el de mayor consumo $57.6 \%$. En relación a otras sustancias están presentes los canabiniodes, consumidos por el $6,3 \%$ de la muestra; la cocaína o un derivado de esta, con un $1,3 \%$ del mismo grupo etario, y los inhalantes, con el $0,5 \%$ de las personas encuestadas entre los 18 y los 44 años.

Así mismo, las cifras de consumo de sustancias entre universitarios colombianos muestran una prevalencia de $7,1 \%$ en el consumo actual de marihuana, mientras que para el caso de «haberla probado», el porcentaje que estimado fue de 22,0\%. La mayor prevalencia de consumo en el último mes se dio en los estratos 1 y 2 , resultados que concuerdan con los estudios realizados por otros investigadores (12).

Cabe resaltar que los datos en 
realizados durante tres periodos específicos: los últimos 30 días, los 12 meses anteriores y alguna vez en la vida, donde los resultados fueron de $23,9 \%, 31,5 \%$ y $39,9 \%$ respectivamente. La sustancia más consumida ha sido siempre la marihuana en todos los casos (20,7\%, 27,4\%, 34,7\%), seguida de LSD $(2,3 \%, 5,5 \%, 6,1 \%)$. En los últimos 12 meses el consumo fue entre 3 a 5 veces en el $24,3 \%$ de los casos (13).

\section{Materiales y métodos}

Se planteó un estudio descriptivo transversal, que permitió identificar la prevalencia del consumo de sustancias en los estudiantes de una facultad del área de la salud (se omite el nombre de la institución por exigencia del Comité de Ética). Para ello, se utilizaron instrumentos de recolección de datos variados; la prevalencia se hizo por medio de encuesta estructurada.

La población estuvo representada por los 537 estudiantes de una facultad del área de la salud, se hizo el cálculo del tamaño muestral teniendo en cuenta los datos de prevalencia de un estudio reciente realizado con estudiantes en la escuela de salud de una universidad privada de Medellín, que fue del (96.2\%) (14). Sin embargo, esta prevalencia por ser tan alta arrojó un tamaño de muestra de 78 estudiantes a encuestar, es por ello que el grupo investigador decidió tomar una prevalencia del $50 \%$ dado que maximiza el tamaño muestral.

Con una confiabilidad del 95\% y un lími-

140 te de confianza inferior de la prevalencia esperada del $50 \%$, se aplicó la fórmula para población finita y con la característica de interés expresada en proporción por Colimon (15) $\mathrm{n}=\mathrm{N}^{\star} \mathrm{z} 2^{\star}$ varianza/ e2(N-1)+(z2* varianza) donde $\mathrm{N}$ : es la población objeto de investigación; $\mathrm{Z}$ : es el percentil de la distribución normal estándar determinado por el nivel de confianza de $95 \%$, esto se representa con un valor de 1,962; e2:está dado por el investigador para calcular la varianza de la muestra; varianza: la cual podrá determinarse en términos de probabilidad de ocurrencia del fenómeno, que se estima sobre marcos de muestreos previos o se define como ocurrió en este cálculo muestral.

Con las condiciones descritas anteriormente el tamaño de la muestra fue de 252 estudiantes, seleccionados en forma aleatoria de la base de datos suministrada por la vicedecanatura de la universidad. La selección de la muestra se realizó mediante muestreo aleatorio estratificado para obtener una muestra con variabilidad similar a la de la población. Los estratos se determinaron por los semestres y el programa que se empleó para este proceso fue Epidat 3.1 utilizado para análisis epidemiológicos de datos tabulados. El procedimiento se realizó por asignación proporcional al tamaño muestral de los diferentes estratos.

Se aplicó una encuesta autodiligencia$\mathrm{da}$, en las cuales se evaluaron variables sociales, demográficas, de consumo y de salud mental. Esta encuesta se compuso de varios instrumentos como el test de DUSI y un cuestionario que valora la motivación para el consumo y los efectos de las sustancias (16). 
El Test de DUSI modificado y adaptado para Colombia consta de preguntas que evalúan aspectos relacionados con la frecuencia, y el uso y abuso de drogas; se clasifica de acuerdo con su puntaje así: riesgo de dependencia a drogas [> 4 puntos], riesgo de dependencia [1-3 puntos] y sin riesgo [0 puntos] $(9,16)$.

La encuesta fue entregada a los estudiantes de cada curso para su autoadministración, solicitando colaboración voluntaria y firma de consentimiento informado; también se aseguró anonimato y confidencialidad. El proceso fue supervisado por los investigadores. Los principios éticos que se tuvieron en cuenta durante el desarrollo del estudio se ajustaron a la normatividad existente para la investigación en seres humanos, Resolución 08430 de 1993 y declaración de Helsinki, entre los cuales están el diseño del consentimiento informado, donde se dio a conocer al participante el objetivo general del proyecto, los métodos para la recolección de datos, las fuentes de financiación, la relación del equipo investigador con la Universidad y los beneficios del estudio. Además, incluyó información sobre la libertad que cada estudiante tenía de diligenciar la encuesta, de retirase, cambiar de opinión en cualquier momento del studio, y/o de decidir que la investigación no concordara con sus intereses o conveniencias.

Con la información recolectada y con la totalidad de los instrumentos diligenciados se elaboró una base de datos en el programas SPSS versión 21 (SPSS Inc.; Chicago, Illinois, USA) con licencia de la Universidad de Antioquia.
Para el proceso de la información, se extrajeron frecuencias absolutas $y$ relativas de las variables nominales; de igual forma se extrajeron las medidas de tendencia central y de dispersión de las variables cuantitativas. Para la recolección de datos se utilizó un instrumento estructurado por los investigadores partiendo de los objetivos y de la operacionalización de las variables, estas son: sociales, demográficas y de consumo.

La investigación fue aprobada por el Comité de Ética de la Facultad de salud de la Universidad, en la cual se realiza el estudio mediante Acta No CEI-FE 2013-14.

\section{Resultados}

\section{Descripción sociodemográfica}

En el estudio participaron 252 estudiantes matriculados en la facultad de salud de una universidad pública de Colombia, de ellos solamente un estudiante se negó a contestar la encuesta. Este estudiante no dio razones para negarse a diligenciar la encuesta.

Las mujeres fueron la población predominante con un $80.2 \%$, donde la edad mínima fue de 18 años y la máxima de 53; la edad de mayor frecuencia fue 21 años con un 13,9\%; la mediana fue de 21,5 , donde el $50 \%$ de la población se ubicó en la edad 22,9 años. La edad mínima de ingreso a la universidad fue de 16 años (4.1\%) y la máxima de 52 años (0.04\%); la edad más frecuente fue de 18 años (25.8\%), seguida de 19 años con (19\%). 
En cuanto al estado civil, predominó el estudiante soltero con el $88,5 \%$, en menor proporción la unión libre con el $6,3 \%$, seguido del casado con $4,4 \%$. Solo una persona manifestó estar separada y no se registraron viudos dentro de la muestra. El estrato socioeconómico más frecuente fue el nivel 3 con el $46 \%$, seguido del estrato 2 con el $38 \%$, el estrato 4 se presentó en un 7,5\% y el estrato de menor proporción fue el 6 con el 0,04\%. En relación a la procedencia el 6,3\% de los participantes ha vivido la mayor parte de su vida en otros departamentos diferentes a Antioquia, como Nariño, Cauca y Valle entre otros. El 3,6\% pertenecen a algún grupo indígena o comunidad negra.

El $72 \%$ manifiestan pertenecer a por lo menos una red social, de ellos el 64,2\% pertenece a redes sociales virtuales como facebook, Twiter y Hotmail, otras redes sociales a las que pertenecen y no hacen parte de la red virtual son grupos juveniles, de danza y natación entre otros.

\section{Descripción de la prevalencia de consumo}

Las sustancias que consumían al momento de la encuesta fueron, en su orden, alcohol en un 75,8\%, tabaco $14,3 \%$, marihuana $6 \%$, cocaína en $1,2 \%$, sintéticas y derivadas de hongos en $0,08 \%$. No se registró consumo de heroína, benzodiacepinas e hipnóticos. El 5,2\% refirió consumo terapéutico de antidepresivos, y sin prescripción médica el 3,6\%. El $53,6 \%$ de los participantes refirió consumo de alguna sustancia antes de haber ingresado a la universidad.

Tabla 1. Descripción del consumo de la población de estudio.

\begin{tabular}{|c|c|c|c|c|c|c|c|c|c|c|}
\hline \multirow[t]{2}{*}{ Variable } & \multicolumn{2}{|c|}{$\begin{array}{l}\text { No consume } \\
(0)\end{array}$} & \multicolumn{2}{|c|}{$\begin{array}{c}\text { Experimental/ } \\
\text { Recreacional } \\
1-2 \text { veces al } \\
\text { mes }\end{array}$} & \multicolumn{2}{|c|}{$\begin{array}{c}\text { Ocasional/ } \\
\text { 3-9 veces en } \\
\text { el mes }\end{array}$} & \multicolumn{2}{|c|}{$\begin{array}{l}\text { Habitual } \\
10-20 \text { veces } \\
\text { en el mes }\end{array}$} & \multicolumn{2}{|c|}{$\begin{array}{c}\text { Compulsivo } \\
\text { Más de } 20 \\
\text { veces en el } \\
\text { mes }\end{array}$} \\
\hline & $\mathrm{Fc}$ & $\%$ & $\mathrm{Fc}$ & $\%$ & $\mathrm{Fc}$ & $\%$ & Fc & $\%$ & $\mathrm{Fc}$ & $\%$ \\
\hline Alcohol & 61 & 24,4 & 139 & 55,2 & 51 & 20,2 & 1 & 0,4 & - & - \\
\hline Cigarrillo & 216 & 85,7 & 9 & 3,6 & 12 & 4,8 & 3 & 1,2 & 12 & 4,8 \\
\hline Éxtasis & 251 & 99,6 & 1 & 0,4 & & - & - & & - & \\
\hline Marihuana & 237 & 94 & 11 & 4,4 & 3 & 1,2 & - & - & 1 & 0,4 \\
\hline Cocaína & 249 & 98,8 & 2 & 0,8 & 1 & 0,4 & - & - & - & - \\
\hline Inhalantes & 251 & 99,6 & - & - & - & - & 1 & 0,4 & - & - \\
\hline \multicolumn{11}{|c|}{ Fármacos auto-medicados (sin prescripción médica) } \\
\hline Antidepresivos & 245 & 97,2 & 6 & 2,4 & 1 & 0,4 & - & & - & \\
\hline
\end{tabular}
puntos]. 


\section{Motivación para el consumo}

Esta descripción de variables hace relación con los motivos relacionados con el consumo de sustancias. En la población participante se encontró que $53,6 \%$ consumió algún tipo de sustancia antes de su ingreso en la universidad, el $56 \%$ de los estudiantes respondió que la mayor motivación para el consumo es por gusto, hubo un 6,7\% que respondió que de alguna manera se ha sentido presionado por los compañeros de estudio para consumir; hubo un $6 \%$ que siente que su familia lo motiva a consumir, el $3 \%$ consume para mejorar el rendimiento académico, hubo un $2,8 \%$ que consume para mejorar el rendimiento sexual, de los que consumen $11,9 \%$ lo hace para matar el tiempo; el 30,6\% de los consumidores dice hacerlo para disipar estados de angustia o estrés y el 11,5\% considera que algunos espacios de la facultad lo motivan a consumir la sustancia.

Efectos de la sustancia y tipos de afección
Estas descripciones hacen relación a los efectos que las sustancias tienen en su vida familiar, social y académica. En este componente se encontró que el $1,6 \%$ manifiesta que bajo el efecto de las sustancias mejora el rendimiento académico, en el $4 \%$ mejora el rendimiento sexual, hubo un $12,7 \%$ expresa sentirse más feliz cuando está bajo el efecto de la sustancia, el 2,0\% dice que mejora su relación con la pareja, el 8,3\% considera que es más tolerante frente a situaciones adversas. Entre las afecciones el 5,2\% incumplió algún encuentro académico por causa del consumo, el 1,6\% ha tenido problemas disciplinarios, el 7,9\% por efectos de la sustancia ha pensado y/o intentado suicidarse, el $16,3 \%$ ha sentido cambios bruscos en el estado de ánimo, el 20,2\% siente que es más arriesgado frente a la toma de decisiones, el 16,3\% le ha molestado alguna vez que los demás lo critiquen por su forma de consumir, el 22,6\% ha tenido la impresión de que debería consumir menos, el 18.3\% alguna vez se ha sentido mal o culpable por el hábito de consumir.

Tabla 2. Características sociodemográficas de los estudiantes relacionado con consumo de alcohol

\begin{tabular}{|c|c|c|c|c|c|c|c|c|}
\hline \multirow{2}{*}{\multicolumn{2}{|c|}{$\begin{array}{c}\text { Variables } \\
\text { sociodemográficas }\end{array}$}} & \multicolumn{7}{|c|}{ Consumo de alcohol } \\
\hline & & \multirow{2}{*}{$\begin{array}{r}\text { No } \\
\text { consume }\end{array}$} & \multirow{2}{*}{\begin{tabular}{r}
\multicolumn{1}{c}{$\begin{array}{c}1-2 \\
\text { veces } \\
\text { al mes }\end{array}$} \\
29
\end{tabular}} & \multirow{2}{*}{\begin{tabular}{r}
\multicolumn{1}{c}{$\begin{array}{c}\text { 3-9 } \\
\text { veces }\end{array}$} \\
al mes \\
13
\end{tabular}} & \multirow{2}{*}{$\begin{array}{l}\begin{array}{l}10-20 \\
\text { veces }\end{array} \\
\text { al mes } \\
0\end{array}$} & \multirow{2}{*}{$\begin{array}{l}\text { Más de } \\
20 \text { veces } \\
\text { al mes } \\
0\end{array}$} & \multirow{3}{*}{$\begin{array}{r}\mathbf{X} 2 \\
4,61\end{array}$} & \multirow{3}{*}{$\begin{array}{l}\mathbf{P} \\
0,33\end{array}$} \\
\hline \multirow{3}{*}{ Sexo } & Hombre & & & & & & & \\
\hline & Mujer & 53 & 101 & 37 & 1 & 1 & & \\
\hline & Primer & 0 & 2 & 0 & 0 & 0 & & \\
\hline \multirow{4}{*}{$\begin{array}{l}\text { Semestre } \\
\text { que } \\
\text { cursa }\end{array}$} & Segundo & 22 & 39 & 9 & 1 & 1 & & \\
\hline & Tercer & 5 & 14 & 10 & 0 & 0 & 21,9 & 0,821 \\
\hline & Cuarto & 6 & 14 & 6 & 0 & 0 & & \\
\hline & Quinto & 5 & 9 & 3 & 0 & 0 & & \\
\hline
\end{tabular}


Continuación Tabla 2. Características sociodemográficas de los estudiantes relacionado con consumo de alcohol

\begin{tabular}{|c|c|c|c|c|c|c|c|c|}
\hline \multirow{2}{*}{\multicolumn{2}{|c|}{$\begin{array}{c}\text { Variables } \\
\text { sociodemográficas }\end{array}$}} & \multicolumn{7}{|c|}{ Consumo de alcohol } \\
\hline & & \multirow{2}{*}{$\begin{array}{c}\begin{array}{c}\text { No } \\
\text { consume }\end{array} \\
0\end{array}$} & \multirow{2}{*}{$\begin{array}{r}\begin{array}{c}1-2 \\
\text { veces } \\
\text { al mes }\end{array} \\
10\end{array}$} & \multirow{2}{*}{$\begin{array}{r}\begin{array}{c}\text { 3-9 } \\
\text { veces } \\
\text { al mes }\end{array} \\
6\end{array}$} & \multirow{2}{*}{ 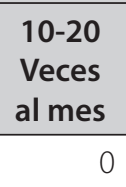 } & \multirow{2}{*}{$\begin{array}{c}\begin{array}{c}\text { Más de } \\
\text { 20Veces } \\
\text { al mes }\end{array} \\
0\end{array}$} & \multirow[t]{2}{*}{$\mathrm{X} 2$} & \multirow[t]{2}{*}{ P } \\
\hline \multirow{4}{*}{$\begin{array}{l}\text { Semestre } \\
\text { que } \\
\text { cursa }\end{array}$} & Sexto & & & & & & & \\
\hline & Séptimo & 3 & 7 & 4 & 0 & 0 & \multirow[t]{2}{*}{21,9} & \multirow[t]{2}{*}{0,821} \\
\hline & Octavo & 19 & 35 & 12 & 0 & 0 & & \\
\hline & Soltero & 49 & 116 & 46 & 1 & 1 & \multirow{5}{*}{7,21} & \multirow{5}{*}{0,969} \\
\hline \multirow{3}{*}{$\begin{array}{l}\text { Estado } \\
\text { civil }\end{array}$} & U. libre & 6 & 7 & 3 & 0 & 0 & & \\
\hline & Casado & 4 & 6 & 1 & 0 & 0 & & \\
\hline & Separado & 1 & 0 & 0 & 0 & 0 & & \\
\hline \multirow{7}{*}{$\begin{array}{l}\text { Estrato } \\
\text { económico }\end{array}$} & Viudo & 0 & 1 & 0 & 0 & 0 & & \\
\hline & 1 & 4 & 8 & 3 & 0 & 0 & \multirow{6}{*}{9,8} & \multirow{6}{*}{0,972} \\
\hline & 2 & 18 & 53 & 21 & 0 & 0 & & \\
\hline & 3 & 31 & 59 & 22 & 1 & 1 & & \\
\hline & 4 & 6 & 8 & 3 & 0 & 0 & & \\
\hline & 5 & 1 & 2 & 0 & 0 & 0 & & \\
\hline & 6 & 0 & 0 & 1 & 0 & 0 & & \\
\hline
\end{tabular}

Se realizó el cruce de variables sociodemográficas con el consumo de alcohol por ser de las sustancias que más se consumían en los estudiantes universi- tarios, en cada uno de los cruces se evidenció que no hay asociación estadísticamente significativa en ninguno de los cruces.

Tabla 3. Características sociodemográficas de los estudiantes relacionado con consumo de cigarrillo

\begin{tabular}{|c|c|c|c|c|c|c|c|c|}
\hline & & \multicolumn{7}{|c|}{ Consumo de cigarrillo } \\
\hline \multicolumn{2}{|c|}{$\begin{array}{c}\text { Variables } \\
\text { sociodemográficas }\end{array}$} & $\begin{array}{c}\text { No } \\
\text { consume }\end{array}$ & $\begin{array}{c}1-2 \\
\text { veces } \\
\text { al mes }\end{array}$ & $\begin{array}{c}\text { 3-9 } \\
\text { veces } \\
\text { al mes }\end{array}$ & $\begin{array}{l}10-20 \\
\text { veces } \\
\text { al mes }\end{array}$ & $\begin{array}{c}\text { Más de } \\
20 \text { veces } \\
\text { al mes }\end{array}$ & $\mathrm{X} 2$ & $P$ \\
\hline \multirow{3}{*}{ Sexo } & Hombre & 36 & 3 & 4 & 2 & 4 & \multirow{3}{*}{11,2} & \multirow{2}{*}{0,047} \\
\hline & Mujer & 172 & 3 & 8 & 1 & 8 & & \\
\hline & Primer & 2 & 0 & 0 & 0 & 0 & & \\
\hline \multirow{4}{*}{$\begin{array}{l}\text { Semestre } \\
\text { que } \\
\text { cursa }\end{array}$} & Segundo & 68 & 1 & 3 & 0 & 0 & & \\
\hline & Tercer & 25 & 1 & 0 & 0 & 3 & 31,58 & 0,634 \\
\hline & Cuarto & 23 & 0 & 2 & 0 & 1 & & \\
\hline & Quinto & 13 & 0 & 1 & 1 & 2 & & \\
\hline
\end{tabular}


Continuación Tabla 3. Características sociodemográficas de los estudiantes relacionado con consumo de cigarrillo

\begin{tabular}{|c|c|c|c|c|c|c|c|c|}
\hline \multirow{2}{*}{\multicolumn{2}{|c|}{$\begin{array}{c}\text { Variables } \\
\text { sociodemográficas }\end{array}$}} & \multicolumn{7}{|c|}{ Consumo de cigarrillo } \\
\hline & & \multirow{2}{*}{\begin{tabular}{r}
\multicolumn{1}{c}{$\begin{array}{c}\text { No } \\
\text { consume }\end{array}$} \\
12
\end{tabular}} & \multirow{2}{*}{\begin{tabular}{r}
\multicolumn{1}{c}{$\begin{array}{c}1-2 \\
\text { veces }\end{array}$} \\
al mes \\
1
\end{tabular}} & \multirow{2}{*}{$\begin{array}{r}\begin{array}{c}\text { 3-9 } \\
\text { veces } \\
\text { al mes }\end{array} \\
1\end{array}$} & \multirow{2}{*}{ 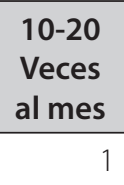 } & \multirow{2}{*}{$\begin{array}{l}\begin{array}{c}\text { Más de } \\
\text { 20Veces } \\
\text { al mes }\end{array} \\
1\end{array}$} & \multirow{2}{*}{$x 2$} & \multirow{2}{*}{$\mathbf{P}$} \\
\hline \multirow{4}{*}{$\begin{array}{l}\text { Semestre } \\
\text { que } \\
\text { cursa }\end{array}$} & Sexto & & & & & & & \\
\hline & Séptimo & 12 & 0 & 1 & 1 & 0 & \multirow[t]{2}{*}{31,58} & \multirow[t]{2}{*}{0,634} \\
\hline & Octavo & 53 & 3 & 4 & 0 & 5 & & \\
\hline & Soltero & 186 & 5 & 12 & 2 & 7 & \multirow{5}{*}{35,24} & \multirow{5}{*}{0,019} \\
\hline \multirow{3}{*}{$\begin{array}{l}\text { Estado } \\
\text { civil }\end{array}$} & U. libre & 13 & 1 & 0 & 0 & 2 & & \\
\hline & Casado & 8 & 0 & 0 & 1 & 2 & & \\
\hline & Separado & 0 & 0 & 0 & 0 & 1 & & \\
\hline \multirow{7}{*}{$\begin{array}{l}\text { Estrato } \\
\text { económico }\end{array}$} & Viudo & 1 & 0 & 0 & 0 & 0 & & \\
\hline & 1 & 14 & 0 & 0 & 0 & 1 & \multirow{6}{*}{46,59} & \multirow{6}{*}{0,005} \\
\hline & 2 & 73 & 1 & 6 & 3 & 8 & & \\
\hline & 3 & 105 & 3 & 4 & 0 & 2 & & \\
\hline & 4 & 14 & 2 & 1 & 0 & 0 & & \\
\hline & 5 & 2 & 0 & 0 & 0 & 1 & & \\
\hline & 6 & 0 & 0 & 1 & 0 & 0 & & \\
\hline
\end{tabular}

Este cruce de variables socio demográficas en relación al consumo de cigarrillo mostró asociación estadística con sexo, estado civil y estrato socioeconómico.

Tabla 4. Características sociodemográficas de los estudiantes relacionadas con consumo de marihuana.

\begin{tabular}{|c|c|c|c|c|c|c|c|c|}
\hline & & \multicolumn{7}{|c|}{ Consumo de marihuana } \\
\hline \multicolumn{2}{|c|}{$\begin{array}{c}\text { Variables } \\
\text { sociodemográficas }\end{array}$} & $\begin{array}{c}\text { No } \\
\text { consume }\end{array}$ & $\begin{array}{c}1-2 \\
\text { veces } \\
\text { al mes }\end{array}$ & $\begin{array}{c}\text { 3-9 } \\
\text { veces } \\
\text { al mes }\end{array}$ & $\begin{array}{c}10-20 \\
\text { veces } \\
\text { al mes }\end{array}$ & $\begin{array}{l}\text { Más de } \\
20 \text { veces } \\
\text { al mes }\end{array}$ & $\mathrm{X} 2$ & $\mathbf{P}$ \\
\hline \multirow{3}{*}{ Sexo } & Hombre & 43 & 6 & 0 & 0 & 0 & \multirow{3}{*}{9,26} & \multirow{2}{*}{0,026} \\
\hline & Mujer & 184 & 5 & 3 & 0 & 1 & & \\
\hline & Primer & 2 & 0 & 0 & 0 & 0 & & \\
\hline \multirow{4}{*}{$\begin{array}{l}\text { Semestre } \\
\text { que } \\
\text { cursa }\end{array}$} & Segundo & 69 & 2 & 0 & 0 & 1 & & \\
\hline & Tercer & 26 & 3 & 1 & 0 & 0 & 22,9 & 0,349 \\
\hline & Cuarto & 21 & 4 & 0 & 0 & 0 & & \\
\hline & Quinto & 16 & 1 & 1 & 0 & 0 & & \\
\hline
\end{tabular}


Continuación Tabla 4. Características sociodemográficas de los estudiantes relacionadas con consumo de marihuana.

\begin{tabular}{|c|c|c|c|c|c|c|c|c|}
\hline \multirow{2}{*}{\multicolumn{2}{|c|}{$\begin{array}{c}\text { Variables } \\
\text { sociodemográficas }\end{array}$}} & \multicolumn{7}{|c|}{ Consumo de marihuana } \\
\hline & & \multirow{2}{*}{$\begin{array}{c}\begin{array}{c}\text { No } \\
\text { consume }\end{array} \\
15\end{array}$} & \multirow{2}{*}{$\begin{array}{r}\begin{array}{c}1-2 \\
\text { veces } \\
\text { al mes }\end{array} \\
0\end{array}$} & \multirow{2}{*}{\begin{tabular}{c|}
$\begin{array}{c}3-9 \\
\text { veces } \\
\text { al mes }\end{array}$ \\
1
\end{tabular}} & \multirow{2}{*}{$\begin{array}{r}\begin{array}{r}10-20 \\
\text { Veces } \\
\text { al mes }\end{array} \\
0\end{array}$} & \multirow{2}{*}{$\begin{array}{r}\begin{array}{c}\text { Más de } \\
\text { 20Veces } \\
\text { al mes }\end{array} \\
0\end{array}$} & \multirow[t]{2}{*}{$\mathrm{X} 2$} & \multirow[t]{2}{*}{$P$} \\
\hline \multirow{4}{*}{$\begin{array}{l}\text { Semestre } \\
\text { que } \\
\text { cursa }\end{array}$} & Sexto & & & & & & & \\
\hline & Séptimo & 13 & 1 & 0 & 0 & 0 & \multirow[t]{2}{*}{22,9} & \multirow[t]{2}{*}{0,349} \\
\hline & Octavo & 65 & 0 & 1 & 0 & 0 & & \\
\hline & Soltero & 199 & 10 & 3 & 0 & 1 & \multirow{5}{*}{1,3} & \multirow{5}{*}{1} \\
\hline \multirow{4}{*}{$\begin{array}{l}\text { Estado } \\
\text { civil }\end{array}$} & U. libre & 15 & 1 & 0 & 0 & 0 & & \\
\hline & Casado & 11 & 0 & 0 & 0 & 0 & & \\
\hline & Separado & 1 & 0 & 0 & 0 & 0 & & \\
\hline & Viudo & 1 & 0 & 0 & 0 & 0 & & \\
\hline \multirow{6}{*}{$\begin{array}{l}\text { Estrato } \\
\text { económico }\end{array}$} & 1 & 15 & 0 & 0 & 0 & 0 & \multirow{6}{*}{34,33} & \multirow{6}{*}{0,003} \\
\hline & 2 & 85 & 4 & 3 & 0 & 0 & & \\
\hline & 3 & 108 & 5 & 0 & 0 & 1 & & \\
\hline & 4 & 17 & 0 & 0 & 0 & 0 & & \\
\hline & 5 & 2 & 1 & 0 & 0 & 0 & & \\
\hline & 6 & 0 & 1 & 0 & 0 & 0 & & \\
\hline
\end{tabular}

Estos cruces evaluaron la asociación de las variables sociodemográficas con el consumo de sustancias como la marihuana, por ser de las sustancias que más se consumían en los estudiantes universitarios; en cada uno de los cruces se evidenció asociación estadística en sexo y estrato socioeconómico.

\section{Discusión}

Este estudio encontró que la sustancia de mayor prevalencia es el alcohol en un $75.6 \%$ en los patrones de consumo experimental y ocasional; por su parte, el cigarrillo se presentó en menor frecuencia,

146 pero con patrón de consumo compulsivo. Estos resultados concuerdan con lo encontrado en estudios con universitarios donde la sustancia más consumida es el alcohol, después el tabaco y de uso ilegal la marihuana $(17,18)$. Si bien la mayor prevalencia de estas sustancias está en los patrones experimental y ocasional, mal llamados consumidores sociales, es importante intervenir la situación porque pueden convertirse en abusadores o dependientes, no es de esperarse esto en estudiantes de una facultad de salud dado que conocen las consecuencias que pueden producir en la salud, no obstante, se minimiza el riesgo de daño y este se sobrepone a la satisfacción de la necesidad que motiva el consumo.

La representación social de una sustancia puede influir en la frecuencia del 
consumo, quizás es por ello que en este estudio se encontró el alcohol como la sustancia de mayor consumo en los estudiantes, no así el tabaco y marihuana debido posiblemente a la molestia social que produce el humo producto de la combustión $(19,20)$. En estudio realizado con adolescentes se explica cómo la concepción social benévola de esta sustancia; el nexo indisociable alcohol-diversión, que la legitima cultural y socialmente; los modelos sociales de consumo, mismos que frecuentemente se encuentran en familiares; y los mensajes contradictorios emitidos por los medios de comunicación, son factores que influyen en el patrón de consumo del alcohol en los adolescentes. Lo anterior, aplica para la población estudiada que se encuentra en adolescencia tardía y adultez temprana. No obstante, la población de este estudio se compone de universitarios de una área de la salud, de quienes se espera mayor conciencia en las consecuencias que esta sustancia tiene para el organismo a corto y largo plazo, y que este conocimiento haya permeado sus decisiones a la hora de consumir, lo que de alguna manera indica que las intervenciones a realizar estén más orientadas al desarrollo de competencias en el cuidado de sí mismos tanto como sujeto como cuidador (21).

En relación a las sustancias que se consideran de mayor poder adictivo, como los inhalantes, la cocaína, la marihuana y las drogas de diseño, los consumos son muy bajos comparados con el alcohol y el cigarrillo (Tabla 1). No se encontró consumo de heroína, metadona ni de benzodiacepinas posi- blemente por tratarse de una facultad de salud y porque su poder adictivo es ampliamente reconocido socialmente; además, porque los consumidores de sustancias como bazuco y sacol son estigmatizados entre los mismos consumidores y emiten expresiones de rechazo como el del 'peor peldaño en la sociedad de consumidores'. Es así como en el estudio realizado por Meza y Furegato (22), se menciona que el fenómeno de las drogas, para las (los) estudiantes del área de la salud, tiene un significado negativo con una visión catastrófica representada en forma de deterioro social, que se relaciona con otros problemas sociales como la prostitución, el desempleo y las personas que habitan las zonas marginales.

Es de resaltar que los resultados obtenidos muestran un comportamiento similar en otros estudios realizados con relación a la edad de inicio de consumo. En este estudio se encontró que el $53.6 \%$ ya había consumido algún tipo de sustancia antes de su ingreso a la universidad, lo que concuerda con el estudio del perfil epidemiológico de la drogadicción en estudiantes universitarios de Ortega, Costa y Vasters (23), que sugiere que al menos el $50 \%$ o más de los jóvenes usuarios de drogas ya había consumido al menos una de estas sustancias antes de ingresar a la universidad.

Los motivos que inducen al consumo de drogas son variados, de manera similar que en otros estudios, en este el $56 \%$ de los consumidores respondió que la mayor motivación para el consumo es por gusto, de igual forma hubo 
un 6.7\% que respondió haberse sentido presionado por los compañeros de estudio para consumir y el $6 \%$ afirma que la familia los motiva a consumir; esto concuerda con un estudio que menciona que las variables significativas fueron también el percibir fácil acceso, el tener amigos, conocidos o familiares que usaron sustancias, la aprobación de los padres y la baja percepción de riesgo (24).

Como se demostró en esta investigación, el riesgo de consumo de sustancias psicoactivas, abarca por lo menos un $75 \%$ de los estudiantes que ya consumen alguna de las sustancias como alcohol, cigarrillo y marihuana. En un gran porcentaje de los casos está asociado a variables sociodemográficas y llama la atención que en relación al consumo de alcohol no se haya encontrado asociación estadística, esto podría tener explicación en la medida que el alcohol es una sustancia licita que se consume de forma esporádica en los adolescentes cuya cantidad y frecuencia va aumentando con el tiempo. En la clasificación de dependencia al alcohol se retoma a Guerri Sierra (25) quien menciona la clasificación de tipo multidimensional, según la cual los pacientes se clasifican en función de la etiología del hábito de consumo y de las consecuencias corporales, psíquicas y sociales derivadas del mismo.

\section{Conclusiones}

Al describir el consumo de sustancias licitas e ilícitas en estudiantes univer-

148 sitarios se pretende evidenciar una problemática de salud que si bien ha sido ampliamente estudiada no se ha profundizado en la manera de desestimular el consumo a edades tempranas, tal y como afirma Hanson (26), quien afirma que las personas que no abusan de la sustancia antes de cumplir los 25 años probablemente no desarrollen un problema relacionado con ellas. La prevención debería ser manejada desde el colegio donde el adolescente necesita reforzar su identidad y donde es más vulnerable al consumo, tanto por la necesidad de experimentar o sentirse bien, como por reducir el estrés o tener la sensación de ser adulto.

Por lo tanto, la prevención del consumo temprano de drogas tanto licitas, alcohol y tabaco, como ilícitas, marihuana, cocaína o éxtasis, entre otras; puede marcar una gran diferencia en la reducción de estos riesgos. Si se puede evitar que los jóvenes experimenten o prueben por curiosidad una droga, es posible retrasar o prevenir la drogadicción.

De igual forma, se puede afirmar que la representación social y la motivación para el consumo de drogas está relacionado con las consecuencias de su práctica, lo que conlleva a daños físicos, mentales y al deterioro de las relaciones sociales y familiares. De igual modo, la necesidad de pertenecer a determinados grupos, la experimentación, la falsa idea de mejorar el rendimiento académico o de mejorar su relación con la pareja, el gusto por determinadas sustancias y la idea de querer evadir una realidad, son los elementos de mayor importancia que justifican el consumo. Existen una serie de significados compartidos que se estructuran como re- 
presentaciones sociales que orientan la conducta de los jóvenes y que otorgan un sentido social dirigido hacia el consumo de drogas.

\section{Referencias}

1. Oficina de las Naciones Unidas contra la Droga y el Delito (UNODC). Informe mundial sobre las drogas (resumen ejecutivo). [Internet]. [Consultado 27 Febrero 2018]. Disponible en: https://www.unodc. org/doc/wdr2016/WDR_2016_ExSum_ spanish.pdf

2. Zapata M., Segura A. Consumo de sustancias psicoactivas en jóvenes escolarizados del departamento de Antioquia, investigación comparativa 2003-2007. Nuevas tendencias y comportamientos sociales asociados. Medellín: Carisma, 2007.

3. Parra A., Peñuela A., Pineda C. Documento propuesta de ajuste de la política nacional de salud mental para Colombia 2014. [Consultado 27 Octubre 2014]. Disponible en: http://www.consejonacionaldetrabajosocial.org.co/cnts/images/ POLITICA-NACIONAL-DE-SALUD-MENTAL-2014.pdf

4. Sarasa A., Sordo L., Molist G., et al. Principales daños sanitarios y sociales relacionados con el consumo de alcohol. Rev. Esp. Salud Publica [Internet]. 2014 ago [Citado 27 febrero 2018] ; 88( 4 ): 469-491. Disponible en: http://scielo. isciii.es/scielo.php?script=sci_arttext\&pi$d=S 1135-57272014000400004 \&$ Ing $=$ es. http://dx.doi.org/10.4321/S113557272014000400004.

5. Gómez M., Castañeda H., Acosta A., et al. Características sociales de salud en urgencias de la Clínica Universitaria Bolivariana (Medellín-Colombia) por intoxicación con sustancias psicoactivas. Investigaciones ANDINA [Internet]. 2015 (septiembre), [Citado 2 marzo 2018]; 17(31): 1408-1417. Disponible en: Fuente Académica Premier.

6. United Nations Research Institute for Social Development (UNRISD). Illicit Drugs: Social Impacts and Policy Responses. [Internet]. Geneva. United Nations Research Institute for social development (UNRISD). 1994. [Consultado 27 octubre 2011]. Disponible en: http://www. unrisd.org/80256B3C005BCCF9/(httpAuxPages)/4C3D0BE90FAD550480256B6400419B57/\%24file/bp2.pdf

7. Grisales C., Toro J. La vivencia universitaria y el consumo de sustancias psicoactivas. Medellín: Fundación Universitaria Luis Amigo; 2009;28.

8. Córdoba E., Betancourth S., Tacán. Consumo de sustancias psicoactivas en una universidad privada de Pasto (Colombia) [Internet]. Psicogente, 2017;20(38):308- 19. [Citado 27 febrero 2018]. Disponible en: http://www.scielo.org.co/scielo.php?script=sci_arttext\&pid=S0124-01372017000200308\&In$\mathrm{g}=e n \& n r m=i s o>$.

9. Larger F., Lope F. Manual de drogodependencia: para profesionales de la salud. Barcelona: liberaría universitaria;2002. p.29-49.

10. Galvis Y., Posada J., Mejía R., et al. Primer Estudio Poblacional de Salud Mental Medellín, 2011-2012. Medellín, ed. Vieco e Hijas; 2012. 219p

11. MINSALUD. Encuesta Nacional de 
12. Martínez J., Arias F., Rodelo A., et al . Prevalencia y factores asociados al consumo de marihuana en estudiantes de 18 a 25 años de una universidad pública, Colombia. Rev Univ. salud. [Internet]. 2016 (diciembre) [Citado 27 febrero 2018]; 18( 3 ): 525-31. Disponible en: http://www.scielo.org.co/scielo.php?script=sci_arttext\&pi$\mathrm{d}=\mathrm{S} 0124-71072016000300012 \& \mathrm{Ing}=\mathrm{en}$. http://dx.doi.org/10.22267/rus.161803.57.

13. Castaño J., García S., Luna J., et al. Estudio de factores asociados y prevalencia de consumo de sustancias psicoactivas ilegales en estudiantes de una universidad colombiana. rev.fac.med. [Internet]. 2017 (marzo) [Citado 27 febrero 2018]; 65(1): 23-30. Disponible en: http://www. scielo.org.co/scielo.php?script=sci_arttext\&pid=S0120-00112017000100023\&Ing=en. http://dx.doi.org/10.15446/revfacmed.v65n1.56471.

14. Tirado A., Álvarez M., Velásquez J., et al. Prevalencia y factores de riesgo para el consumo y dependencia de drogas en estudiantes de una universidad de Medellín, Colombia, 2009. Revista Facultad Nacional de Salud Pública 2012;30(1):3844.

15. Colimon KM. Fundamentos de Epidemiologia. $3^{a}$ ed. Medellín: CIB (Corporación para Investigaciones Biológicas). 2010. p. $176-77$

16. Lopera J., Rodríguez M., Mejía R. Validación de la Escala Dusi R-Abreviada, para tamizaje del riesgo de consumo de sustancias en jóvenes de Medellín, Colombia 1999. Intersalud. [Comunicación presentada en: 1er. Congreso Virtual de Psiquiatría. Interpsiquis 2000. Psiquiatria.com. Febrero-Marzo de 2000]. 2000.

17. Calderón E., Cáliz N. Juventud y universidad: sujetos y escenarios para el debate crítico y autorreflexivo sobre el consumo de sustancias psicoactivas de uso legal e ilegal. Rev. Gerenc. Polít.

150 Salud. 2015;14(28):123-41. http://dx.doi. org/10.11144/Javeriana.rgyps18-28.juse
18. Vargas Á., Londoño N. Creencias asociadas al consumo y dependencia de sustancias psicoactivas. Revista Katharsis [Internet]. 2016 (enero), [Cited 2 marzo 2018]; (21): 111-130.

19. Gómez M., Castañeda H., Acosta A., et al. Características sociales de salud en urgencias de la Clínica Universitaria Bolivariana (Medellín-Colombia) por intoxicación con sustancias psicoactivas. Investigaciones ANDINA [Internet]. 2015(septiembre), [Citado 2 marzo 2018]; 17(31):1408-17.

20. Córdoba E., Betancourth S., Tacán L. Consumo de sustancias psicoactivas en una universidad privada de Pasto, Colombia. Psicogente [Internet]. 2017 (julio), [Citado 2 marzo 2018]; 20(38): 308-19.

21. Musitu G. ¿Por qué los adolescentes tienen una baja percepción de riesgo respecto del consumo de alcohol? La visión de los expertos. Metamorfosis [Internet]. 2016 p. 55-73. Disponible en: http://revistametamorfosis.es/index.php/ metamorfosis/article/view/21

22. Meza M., Furegato A. Experiencias y significados Sobre el fenómeno de Las Drogas en Estudiantes de Enfermería. Rev. Latino-Am. Enfermería [Internet]. 2011 junio [Citado 3 junio 2014]; 19(SPE):691-698. Disponible en: http:// www.scielo.br/scielo.php?script=sci_arttext\&pid=S0104-11692011000700005\&I$\mathrm{ng}=\mathrm{en}$.

23. Ortega C., Costa M., Vasters G. Perfil epidemiológico da toxicodependência em Estudiantes Universitarios. Rev. Latino-Am. Enfermería [Internet]. 2011 junio [Citado 27 junio 2014]; 19(SPE):665672. Disponible en: http://www. scielo.br/scielo.php?script=sci_arttex$\mathrm{t} \& \mathrm{pid}=\mathrm{S} 0104-11692011000700002 \& \mathrm{In}-$ $\mathrm{g}=\mathrm{en}$.

24. Osorio A., Ortega M., Pillon S. Factores de riesgo asociados al uso de drogas en estudiantes adolescentes. 
Rev. Latino-Am. Enfermería [Internet]. 2004 abril [Citado 27 junio 2014]; 12(SPE):369-375. Disponible en: http:// www.scielo.br/scielo.php?script=sci_arttext\&pid=S0104-11692004000700011\&lng=en. http://dx.doi.org/10.1590/S010411692004000700011
25. Guerri S. Alcohol. En: Tratado SET de Trastornos adictivos. Madrid: editorial medica panamericana. 2006. p. $153-180$.

26. Hanson G. New vistas in drug abuse prevention. NIDA Research Findings. 2002;16(6). 
INVESTIGACIONES ANDINA No. 35, Vol. 19 